\title{
Wide wavelength tuning of an optical parametric oscillator through electro-optic shaping of the gain spectrum
}

\author{
P. Gross, M. E. Klein, H. Ridderbusch, D.-H. Lee, J.-P. Meyn, and R. Wallenstein \\ Fachbereich Physik, Universität Kaiserslautern, 67663 Kaiserlautern, Germany \\ K.-J. Boller \\ Faculty of Applied Physics, University of Twente, P.O. Box 217, 7500 AE Enschede, The Netherlands
}

Received February 8, 2002

\begin{abstract}
We present a novel technique for tuning an optical parametric oscillator (OPO) through electro-optically induced shape variations of the parametric gain spectrum in quasi-phase-matched lithium niobate (LN). The diode-pumped, triply resonant continuous-wave OPO is based on a 58-mm-long LN crystal that consists of three equally long sections, the outer sections being periodically poled with a $50 \%$ duty cycle. The center section is single-domain material and serves as a tunable phase shifter through the electro-optic effect. By application of a voltage of up to $1230 \mathrm{~V}$, the OPO signal and idler wavelengths are tuned over $102 \mathrm{~nm}$ from 1560 to $1660 \mathrm{~nm}$, in good agreement with theory. (C) 2002 Optical Society of America
\end{abstract}

OCIS codes: $190.2620,190.4360,190.4970,230.2090$.

Continuous-wave (cw) optical parametric oscillators (OPOs) based on periodically poled materials have become mature sources for generating wavelength-tunable, coherent near- and mid-infrared radiation. ${ }^{1,2}$ With periodically poled $\mathrm{LiNbO}_{3}$ (PPLN), a wide wavelength range from 330 to $5500 \mathrm{~nm}$ can be covered, which is usually achieved with a set of differently poled crystals in combination with changing the crystal temperature. ${ }^{3}$ A disadvantage, particularly of temperature tuning, is that the tuning is slow.

In this Letter we demonstrate a different tuning method offering much faster wavelength access. Our OPO tuning is based on the electro-optic effect in a segmented lithium niobate (LN) crystal, which modifies the spectral shape of the parametric gain and thereby shifts the gain maximum and the oscillation wavelength. The OPO was tuned from 1560 to $1610 \mathrm{~nm}$ (signal wave) and from 1610 to $1660 \mathrm{~nm}$ (idler), which is to our knowledge the widest electro-optic tuning of an OPO reported so far. We note that our approach is different from that for a previously reported OPO, where electro-optic tuning was based on asymmetrically poled PPLN with a $75 \%$ duty cycle ${ }^{4}$ which resulted in a smaller tuning range of $\sim 1 \mathrm{~nm}$.

To illustrate the working principle of our OPO tuning, we consider the segmented LN crystal shown in Fig. 1, which we prepared by electric field poling. The crystal consists of three segments of equal length $L_{C} / 3$, where the first and the last segments are poled with a period of $\Lambda$ and a $50 \%$ duty cycle. The spatial periodicity of $\chi^{(2)}$ of the first segment is manufactured to be spatially in phase with that of the last segment. The center segment consists of single-domain $\mathrm{LiNbO}_{3}$ and carries a pair of electrodes. A voltage $U$ can be applied across the crystal thickness $d$ to electrooptically change the refractive index and dispersion in that segment.

Considering the case of a single-pass parametric process, we note that the signal wave experiences a sinc-shaped gain in each of the two outer periodically poled segments. In the center segment, when an electric field $E=U / d$ is applied, the pump, signal, and idler waves acquire an electro-optically induced phase shift with respect to one another. The gain profile in the last crystal segment is phase shifted compared with that of the first segment. With proper adjustment of this phase shift through the applied voltage, the last segment continues to enhance the signal wave and adds to the gain of the first segment. However, if the last segment is adjusted to be out of phase with the first one, backconversion of the output waves into the pump wave takes place, which results in a reduced or even zero total gain for a given signal wavelength. To calculate the parametric small-signal gain for a single pass through the crystal, we use the approach described in Ref. 5 for second-harmonic generation. The generated gain at the signal wavelength can be derived to be

$$
g \propto \operatorname{sinc}^{2}\left(\frac{\Delta k \frac{L_{C}}{3}}{2}\right) \cos ^{2}\left[\frac{\Delta \Phi}{2}+2\left(\frac{\Delta k \frac{L_{C}}{3}}{2}\right)\right] .
$$

$\Delta k$ is the wave-vector mismatch in the first and last segments, given by $\Delta k=2 \pi\left(n_{3} / \lambda_{3}-n_{1} / \lambda_{1}-\right.$ $\left.n_{2} / \lambda_{2}-1 / \Lambda\right)$ with refractive indices $n_{1,2,3}$ and vacuum wavelengths $\lambda_{1,2,3}$, where the subscripts 1,2 , and 3 refer to the idler, signal, and pump wave, respectively. $\Lambda$ is the domain-inversion grating

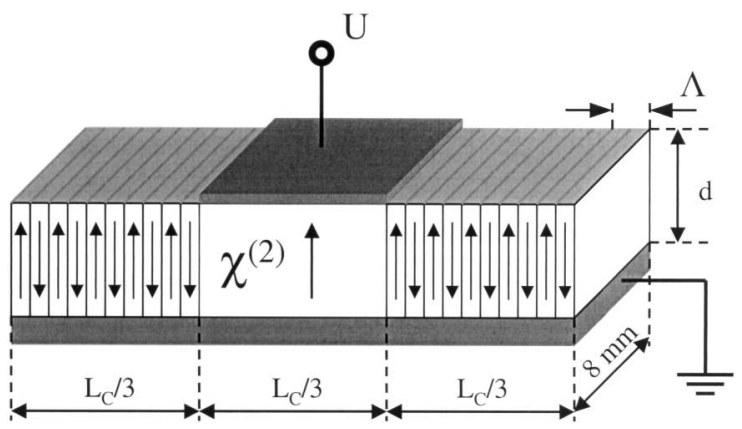

Fig. 1. Schematic drawing of a segmented LN crystal. The arrows indicate the direction of the $\chi^{(2)}$ nonlinear coefficient. 
period, and $\Delta \Phi$ is the electro-optically induced phase shift, $\Delta \Phi=2 \pi\left(\Delta n_{3} / \lambda_{3}-\Delta n_{1} / \lambda_{1}-\Delta n_{2} / \lambda_{2}\right) L_{C} / 3$, where $\Delta n_{1,2,3}=-1 / 2 n_{1,2,3}^{3} E r$ is the change of the refractive index induced by the applied field $E$ and $r$ is the effective electro-optic coefficient of the crystal material. ${ }^{6,7}$ The total gain is a function of the wavelengths, the crystal temperature, and the electric field. Because of the $\cos ^{2}$ dependence in expression (1), one expects the parametric gain to change with a periodicity of the electro-optically induced phase shift of $\Delta \Phi / 2=\pi$. Using expression (1), we calculate the parametric gain spectrum for the signal and idler waves as a function of the crystal temperature and the applied electric field and compare the results with the experimental results below.

The experimental setup is shown in Fig. 2. As a pump source we use a grating-stabilized, singlefrequency, single-stripe diode laser (SDL 5410) in Littman configuration. After transmission through an electro-optic modulator and a $60-\mathrm{dB}$ optical isolator, a nearly diffraction-limited output of up to $14 \mathrm{~mW}$ is available. The wavelength is tunable over $10 \mathrm{~nm}$ near $805 \mathrm{~nm}$. The beam is spatially mode matched to the fundamental mode of the OPO cavity by a spherical lens of $100-\mathrm{mm}$ focal length, which generates a measured beam waist of $35 \mu \mathrm{m}$.

The triply resonant OPO has a linear two-mirror cavity, where both mirrors have a radius of curvature of $50 \mathrm{~mm}$ and are $125 \mathrm{~mm}$ apart, resulting in a beam waist of $40 \mu \mathrm{m}$ for the fundamental cavity mode. To adjust the cavity length, we mount output mirror M2 on a piezo transducer. The pump input mirror, M1, is coated for power transmission of $4 \%$ at the pump wavelength and for broadband high reflectivity at the signal and idler wavelengths, i.e., $R>99.8 \%$ from 1530 to $1730 \mathrm{~nm}$. The second mirror, M2, is broadband highly reflective for the pump, signal, and idler wavelengths $(R>99.8 \%)$.

The 58-mm-long segmented crystal is made from 0.5-mm-thick LN with broadband antireflection-coated faces for the pump, signal, and idler waves. The first and the third 19-mm-long segments are periodically poled with the same domain grating periods in the direction of beam propagation (see Fig. 1). In the transverse dimension of the 8-mm-wide crystal, four channels, with gratings periods of 20.2, 20.4, 20.6, and $20.8 \mu \mathrm{m}$, are available. An electrode of conductive silver lacquer is applied to the single-domain center segment on one side of the crystal. The other electrode is formed by a brass plate attached to the opposite side of the crystal over its entire length. Via the electrodes, a voltage ranging from -1350 to $+1350 \mathrm{~V}$ can be applied across the center segment. By use of an oven, the crystal temperature can be set to values of up to $200{ }^{\circ} \mathrm{C}$ with an estimated stability of $10 \mathrm{mK}$.

$\mathrm{Cw}$ operation of the OPO is obtained by electronic stabilization of the cavity length to the pump wavelength with the piezo transducer by use of the Pound-Drever-Hall (PDH) technique. ${ }^{8}$ The servo bandwidth of the PDH loop is limited to $\sim 500 \mathrm{~Hz}$ by the piezo transducer in combination with the high-voltage amplifier. The OPO provides a low threshold pump power of $600 \mu \mathrm{W}$, and the maximum OPO output power (signal plus idler, measured behind M2) is $47 \mu \mathrm{W}$ at a pump power of $13.5 \mathrm{~mW}$. We note that, with an output mirror M2 with $4 \%$ transmission of the signal and the idler waves, an output power of $2.1 \mathrm{~mW}$ is generated at $13.8-\mathrm{mW}$ pump power, but the threshold pump power increases to $4.5 \mathrm{~mW}$ accordingly.

To verify the temperature dependence of the parametric gain spectrum, as given by expression (1), we measure the OPO wavelengths as a function of the crystal temperature with a monochromator (Oriel; resolution, $0.1 \mathrm{~nm}$ ). The pump wavelength is $804.4 \mathrm{~nm}$, and the domain grating period is $20.2 \mu \mathrm{m}$. The measured OPO output wavelengths are shown in Fig. 3. The signal and idler wavelengths are tuned in the range from 1460 to $1790 \mathrm{~nm}$ if the crystal temperature is varied from 135 to $158^{\circ} \mathrm{C}$. At signal wavelengths below $1460 \mathrm{~nm}$, the OPO ceases to oscillate as a result of increasing losses of the crystal coatings. The signal and idler wavelengths become degenerate at $1608.8 \mathrm{~nm}$ for crystal temperatures of $135.2,138.4$, 139.7 , and $141.3^{\circ} \mathrm{C}$. Increasing the temperature from any of these values results in a smooth decrease of the signal wavelength and a smooth increase of the idler wavelength.

To calculate the theoretically expected OPO tuning behavior, we assume that the OPO will always oscillate at the signal wavelength at which the spectral gain profile of the crystal has an absolute maximum. For each temperature, we calculated the signal gain spectrum according to expression (1), using the Sellmeier coefficients given in Ref. 9, and the electro-optic coefficient $r_{33}=34 \mathrm{pm} / \mathrm{V}{ }^{10}$ The solid curves in Fig. 3 represent the signal and idler wavelengths of the parametric gain maximum for each crystal temperature. Using a constant temperature offset of $23.3^{\circ} \mathrm{C}$ and a constant voltage offset of $500 \mathrm{~V}$ as the only fitting parameters, our calculations show good agreement with the experimental data. The temperature offset can be explained readily by slight errors in the Sellmeier coefficients, as was also found in earlier experiments with PPLN OPOs. ${ }^{2}$ The voltage offset is comparable to what has been observed in second-harmonic experiments in segmented $\mathrm{LiNbO}_{3}$ crystals. ${ }^{11}$

For the central experiments on electro-optic tuning, a varying voltage is applied to the crystal, while all other parameters are held constant (pump wavelength, $806.6 \mathrm{~nm}$; crystal temperature, $57.0^{\circ} \mathrm{C}$; domain grating period, $20.6 \mu \mathrm{m}$ ). The measured OPO wavelengths are shown in Fig. 4. Electro-optic

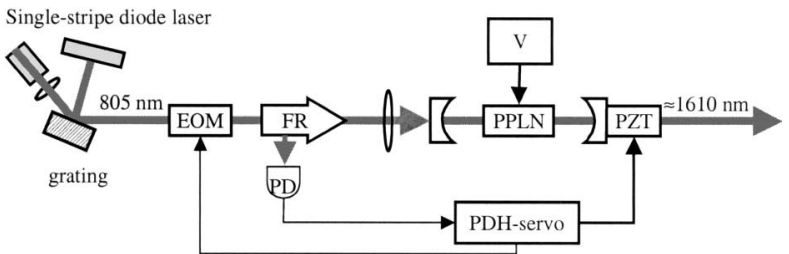

Fig. 2. Experimental setup of the cw OPO with a segmented LN crystal: EOM, electro-optic modulator; FR, optical isolator; PD, photodiode; PZT, piezo transducer; V, voltage supply. 


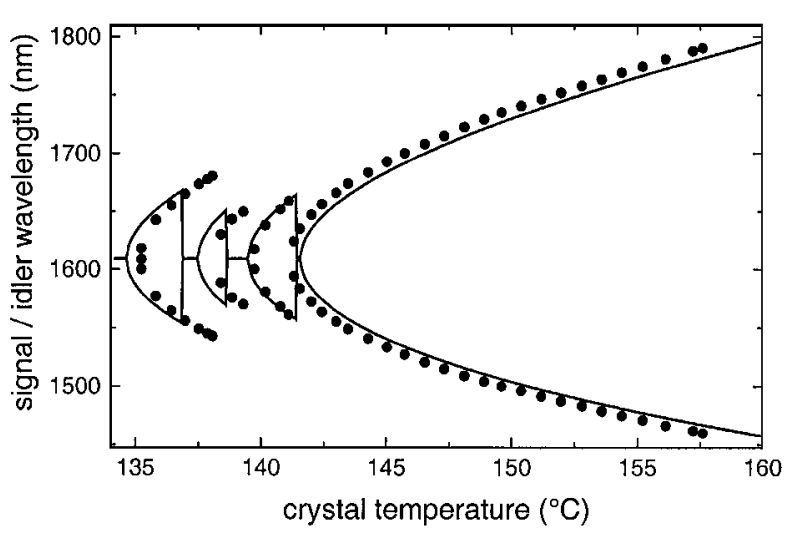

Fig. 3. Signal and idler wavelengths as a function of crystal temperature. Pump wavelength, $804.4 \mathrm{~nm}$; grating period, $20.2 \mu \mathrm{m}$; no voltage applied. Filled circles, experimental data; solid curves, theoretical calculations.

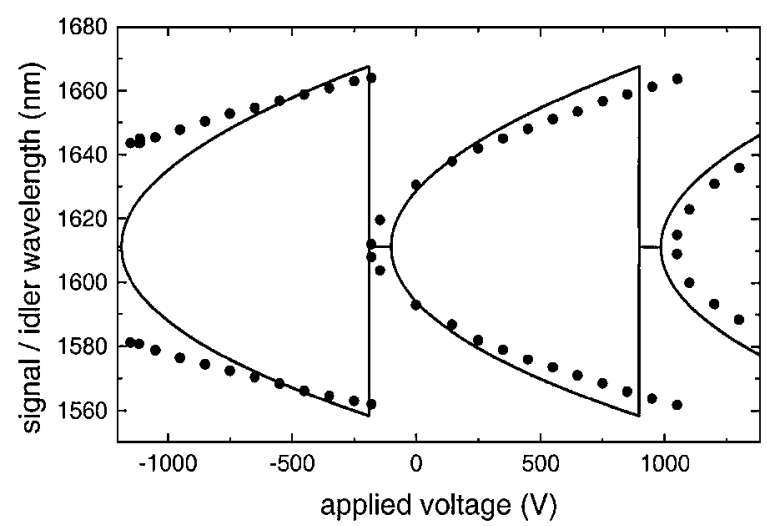

Fig. 4. Signal and idler wavelengths as a function of applied voltage. Crystal temperature, $57.0^{\circ} \mathrm{C}$; pump wavelength, $806.6 \mathrm{~nm}$; grating period, $20.6 \mu \mathrm{m}$. Filled circles, experimental data; solid curves theoretical calculations.

tuning gives access to wide wavelength coverage of $102 \mathrm{~nm}$, i.e., from 1562 to $1613 \mathrm{~nm}$ for the signal wave, and from 1613 to $1664 \mathrm{~nm}$ for the idler wave, for an applied voltage from -180 to $+1050 \mathrm{~V}$. It can also be seen that the OPO wavelengths vary periodically with the voltage, as expected from the $\cos ^{2}$ dependence of expression (1) from the electro-optically induced phase shift, $\Delta \Phi$. Specifically, the observed period of $1230 \mathrm{~V}$ in Fig. 4 should correspond to a phase shift of $\Delta \Phi / 2=\pi$, i.e., to a full cycle of the $\cos ^{2}$ oscillation. The solid curves show the theoretically expected electro-optic tuning. As before, we calculate the signal-wavelength spectral gain profile for each applied voltage to determine the signal wavelength of maximum gain. The theoretical values are shifted to lower temperatures by a constant offset of $21.7^{\circ} \mathrm{C}$, and the voltage offset is $310 \mathrm{~V}$. The experimental data show good agreement with the theoretical prediction.

A main motivation for investigating an electrooptically tunable OPO is the possibility of rapid wavelength tuning. To tune the OPO wavelengths with a defined speed, we apply a time-varying voltage to the electrodes. To monitor the OPO spectrum online, we use a home-built spectrum analyzer, where the OPO output is sent on to a grating (1200 lines $/ \mathrm{mm}$ ) and where the first diffraction order is focused on a linear GaAs CCD camera. Although the spectral resolution is only moderate $(0.4 \mathrm{~nm})$, the advantage of this setup is that one can simultaneously detect an oscillation at any wavelength within a wide spectral range of $\sim 100 \mathrm{~nm}$ around degeneracy.

We apply a periodic (triangular) voltage with $513-\mathrm{V}$ amplitude and a frequency of $0.11 \mathrm{~Hz}$. The signal wave is tuned within $4.6 \mathrm{~s}$ over $9.7 \mathrm{~nm}$ (centered around $1598 \mathrm{~nm}$ ), and the idler wave is tuned over $10.8 \mathrm{~nm}$, while the PDH loop maintains $\mathrm{cw}$ operation. Limited by the resolution of the spectrum analyzer, we can show that every signal and idler wavelength within the scanned interval is generated. The tuning speed of $0.11 \mathrm{~Hz}$ is limited by the PDH servo bandwidth. Further improvement of the demonstrated technique for complete exploitation of the potential of rapid wavelength tuning with the electro-optic effect would require a PDH loop with higher speed or a OPO without pump enhancement.

In summary, we have demonstrated a novel method of electro-optic tuning of the output wavelengths of a OPO. For a first investigation of this method we used a triply resonant cw OPO based on a segmented crystal of PPLN pumped by a single-stripe diode laser emitting near $805 \mathrm{~nm}$. By application of a voltage to the single-domain center segment of the crystal, the signal and idler wavelengths are tuned within a wide range of $102 \mathrm{~nm}$ around degeneracy. We believe this to be the widest electro-optic wavelength tuning of a OPO so far. The presented method offers the possibility of rapid wavelength tuning, such that OPO sources using the presented tuning method in segmented crystals might find applications as highly wavelength-agile injection seeders, e.g., in light detection and ranging (lidar) systems based on high-power pulsed OPOs. ${ }^{12}$

\section{References}

1. L. E. Myers, R. C. Eckardt, M. M. Fejer, R. L. Byer, W. R. Bosenberg, and J. W. Pierce, J. Opt. Soc. Am. B 12, 2102 (1995).

2. M. E. Klein, D.-H. Lee, J.-P. Meyn, K.-J. Boller, and R. Wallenstein, Opt. Lett. 24, 1142 (1999).

3. R. L. Sutherland, Handbook of Nonlinear Optics (Marcel Dekker, New York, 1996), Chap. 3.

4. N. O'Brien, M. Missey, P. Powers, and V. Dominic, Opt. Lett. 24, 1750 (1999).

5. M. M. Fejer, G. A. Magel, G. H. Jundt, and R. L. Byer, IEEE J. Quantum Electron. 28, 2631 (1992).

6. J. F. Nye, Physical Properties of Crystals (Oxford U. Press, Oxford, 1957), Chap. 13.

7. We note that there is an additional phase shift caused by the reverse piezoelectric effect. As this effect is smaller than the electro-optic effect by approximately 3 orders of magnitude, we can neglect it.

8. R. W. P. Drever, J. L. Hall, F. V. Kowalski, J. Hough, G. M. Ford, A. J. Munley, and H. Ward, Appl. Phys. B 31, 97 (1983).

9. D. H. Jundt, Opt. Lett. 22, 1553 (1997).

10. R. S. Weis and T. K. Gaylord, Appl. Phys. A 37, 191 (1985).

11. H. Ridderbusch, M. E. Klein, P. Gross, D.-H. Lee, J.-P. Meyn, R. Wallenstein, and K.-J. Boller, J. Opt. Soc. Am. B 19, 280 (2002).

12. S. T. Yang and S. P. Velsko, Opt. Lett. 24, 133 (1999). 